\title{
DIAGNÓSTICO DE ESTENOSE ESOFÁGICA PARCIAL EM UM CÃO
}

\author{
RAMOS, Mayara Cristtine ${ }^{1}$; \\ NOVO, Tatiane Tavares Schimitt ${ }^{1}$; \\ CAVALCANTI, Eduarda Aléxia Nunes Louzada Dias ${ }^{1}$; \\ BOANOVA, Heloisa do Amaral ${ }^{1}$; \\ CAVALCANTI, Guilherme Albuquerque de Oliveira ${ }^{1}$.
}

\section{RESUMO}

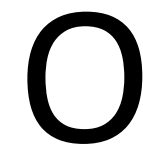
megaesôfago é definido por uma dilatação do esôfago. Essa afecção pode ser classificada em congênita, idiopática, adquirida ou secundária. O megaesôfago secundário à estenose é caracterizado pela obstrução parcial ou total do esôfago, impedindo a passagem do conteúdo alimentar até o estômago, provocando regurgitação e perda de peso no animal. Foi atendida uma cadela com seis anos de idade, sem raça definida, com histórico de regurgitação. No exame físico não foram constatadas alterações. O animal foi submetido a exame de endoscopia e exames radiográficos simples e contrastado. No exame de endoscopia foi visualizada constrição irregular do lúmen. $\mathrm{Na}$ radiografia simples não foram observadas alterações, porém na radiografia contrastada com alimento observou-se estenose parcial e megaesôfago. Com base nos achados imaginológicos, o animal foi diagnosticado com megaesôfago secundário à estenose esofágica parcial e procedeu-se tratamento clínico de manejo alimentar.

Palavras-chave: Megaesôfago. Radiografia. Cães. 


\section{INTRODUÇÃO}

Megaesôfago é uma doença caracterizada por dilatação esofágica, resultante de esôfago aperistáltico, secundário a distúrbio neuromuscular (TILLEY; SMITH, 2003). Pode ser um distúrbio primário (congênito, idiopático ou adquirido) ou secundário (HAN, 2003).

Dentre as estenoses adquiridas, têm-se as estenoses esofágicas que ocorrem quando há fibrose pós esofagite severa que envolve a submucosa e a túnica muscular, podendo obstruir parcial ou totalmente a passagem do conteúdo alimentar até o estômago (ADAMAMAMORAITOU et al., 2002). Os sinais clínicos da doença são regurgitação, perda de peso ou crescimento deficiente, hipersalivação e som de borbulhas a deglutição (TANAKA et al., 2010).

Para o diagnóstico das estenoses esofágicas podem ser utilizadas as radiografias simples, radiografias contrastadas e a fluoroscopia, porém apenas as duas primeiras são de amplo acesso para a classe veterinária. Dentre os exames radiográficos, as radiografias simples e as contrastadas com sulfato de bário líquido não diagnosticam as estenoses esofágicas parciais em cães e o clínico tem que estar ciente das manobras diagnósticas para esses casos, como o exame endoscópico e a radiografia contrastada com alimento (BRAWNER; BARTELS, 1983; LAM et al., 2013).

Já que o tratamento correto da estenose esofágica parcial causadora de megaesôfago depende de um diagnóstico bem realizado, objetiva-se relatar um caso raro e sem descrição da técnica utilizada na literatura especializada nacional, realizado por meio de radiografia torácica.

\section{RELATO DE CASO}

Uma cadela com seis anos de idade e $25,9 \mathrm{~kg}$ foi atendida no Hospital de Clínicas Veterinárias da Universidade Federal de Pelotas (HCV/UFPEL) com histórico de regurgitação há dois anos, já tendo sido consultada por outros profissionais veterinários. A tutora relatou que houve piora no estado de regurgitação da paciente há sete dias, razão pela qual a trouxe para nova consulta. 
O animal apresentou-se sem alterações significativas no exame físico geral e hematologia laboratorial. A seguir, foram realizados exames de radiografia simples, radiografia contrastada, exame de endoscopia e exame de radiografia contrastada com alimento sólido, para auxiliar no diagnóstico. Na radiografia contrastada, utilizou-se sulfato de bário líquido (Figura 1) administrado na dose de $6 \mathrm{~mL} / \mathrm{kg}$ por via oral. Posteriormente, para a segunda radiografia contrastada, foi utilizado alimento sólido misturado ao sulfato de bário, realizouse esse exame utilizando a ração do paciente e o conteúdo de um sachê de ração para gatos, adicionados de $5 \mathrm{~mL} / \mathrm{kg}$ de bário, prontamente ingeridos pelo animal.

Recomendou-se encaminhamento a procedimento cirúrgico, porém a tutora preferiu, primeiramente, realizar o tratamento clínico de administração de alimentação pastosa fracionada em três a quatro vezes ao dia, evitando todo o tipo de alimento sólido para não lesionar ainda mais a musculatura lisa do esôfago. $\mathrm{O}$ animal adaptou-se bem a alimentação pastosa e a tutora não submeteu a cadela ao procedimento cirúrgico.

\section{RESULTADOS E DISCUSSÃO}

O paciente apresentou o que foi descrito por Brawner e Bartels (1983) como o sinal clínico mais frequente do megaesôfago: a regurgitação, que pode ocorrer imediatamente no período pós-prandial ou horas após a ingestão do alimento ou de água. O mesmo sinal também foi observado por Andrade (2007), Souza et al. (2007) e Teófilo et al. (2016).

O diagnóstico de megaesôfago, seja ele idiopático ou secundário a doenças sistêmicas (como botulismo e miastenia gravis) ou por constrição por massas, esofagites, ou persistência de anel vascular, é obtido através da associação do histórico, exame clínico e exames de imagem, principalmente exames radiográficos simples e contrastado (BRAWNER; BARTELS, 1983; LAM et al., 2013).

O exame radiográfico é um método de diagnóstico rápido, de baixo custo e fácil acesso, amplamente utilizado na avaliação de megaesôfago em cães (TANAKA et al., 2010). Pode detectar o megaesôfago por aumento de volume do órgão devido a gás ou alimento, aumento da radiopacidade na face ventral das vértebras torácicas de T1 a T5 e alteração da conformação da traqueia. Usualmente, para confirmação, utiliza-se o exame contrastado com sulfato de bário líquido, este, por sua vez, demonstraria a alteração no volume do órgão 
(BRAWNER; BARTELS, 1983). No presente relato, ambos os exames resultaram inconclusivos, como mostrado na Figura 1.

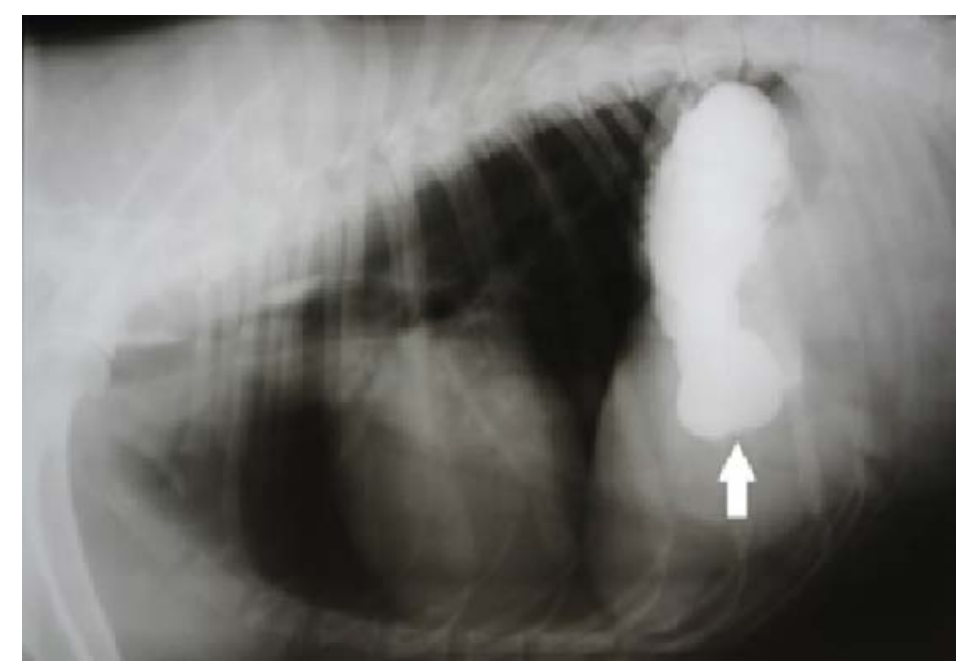

Figura 1 - Presença de sulfato de bário líquido (seta branca) no estômago, imediatamente após sua ingestão.

Além do exame radiográfico, o exame endoscópico vem sendo cada vez mais utilizado na avaliação esofágica, sendo frequentemente realizado após a avaliação radiográfica simples, adicionando informações aos exames já realizados (LAM et al., 2013). Neste caso, foi auxiliar, por demonstrar uma constrição irregular no terço final do esôfago (Figura 2) (HAN, 2003; TANAKA et al., 2010).

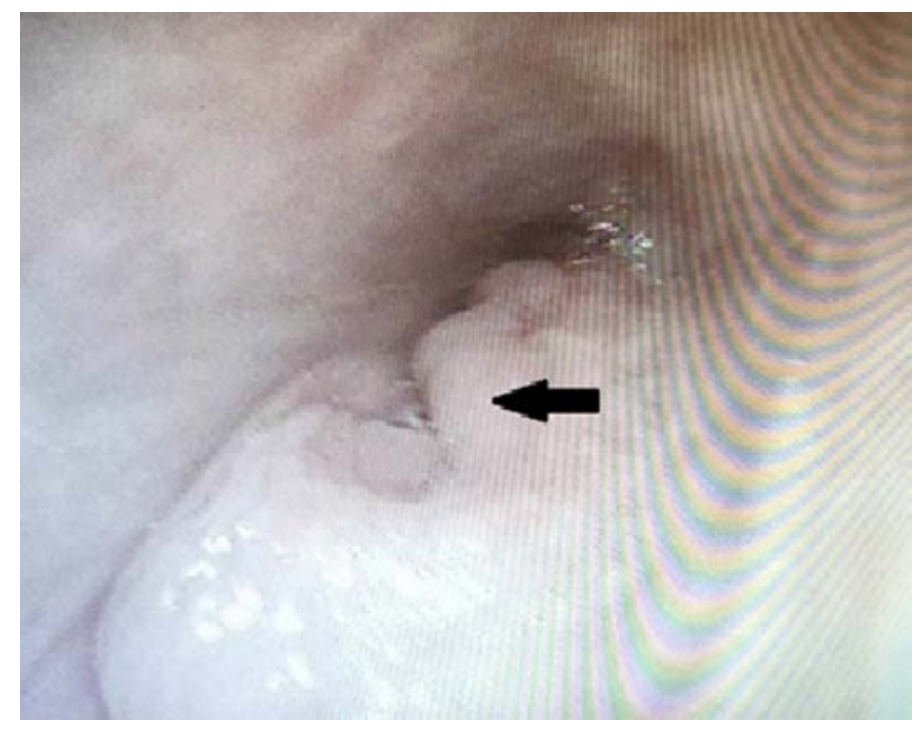

Figura 2 - Esôfago no exame endoscópico mostrando constrição irregular no terço final do órgão. 
O uso de radiografia contrastada com alimento sólido deve ser realizado nos casos em que os outros exames tenham resultado em diagnóstico inconclusivo. (BRAWNER; BARTELS, 1983). No presente relato, o megaesôfago e a localização exata da constrição foram prontamente identificados com este exame (Figura 3), sendo de fundamental importância para a definição do diagnóstico.

No megaesôfago secundário, a melhora do quadro é obtida por dilatações por balões, uso de stents e ressecções da região estenosada (LAM et al., 2013). No caso descrito, foi recomendada a intervenção cirúrgica para a correção da estenose, porém a tutora preferiu fazer o manejo alimentar do animal, já que nenhum método cirúrgico melhora a motilidade no megaesôfago crônico, como definido por Tanaka et al. (2010).

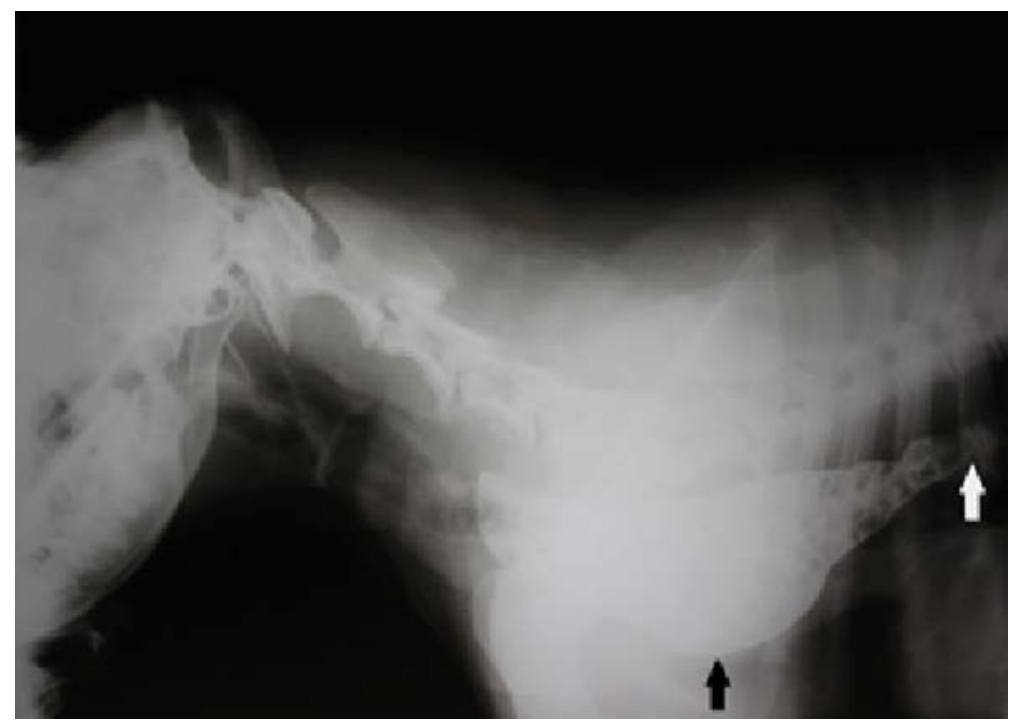

Figura 3 - Radiografia cervical contrastada com alimento sólido em projeção latero-lateral, evidenciando megaesôfago (seta preta) e o local da estenose esofágica (seta branca).

\section{CONCLUSÃO}

O diagnóstico de megaesôfago secundário a estenose pode representar um desafio para os clínicos e imaginologistas veterinários, portanto diferentes técnicas radiográficas e a endoscopia podem ser utilizadas. 


\section{DIAGNOSIS OF PARTIAL ESOPHAGEAL STENOSIS IN A DOG}

\section{ABSTRACT}

M

egaesophagus is defined by dilatation of the esophagus. This condition can be classified as congenital, idiopathic, acquired or secondary. Megaesophagus secondary to stenosis is characterized by partial or complete obstruction of the esophagus, preventing the passage of food to the stomach. It causes regurgitation and weight loss to the animal. A six-year-old female dog, with no defined breed, with a history of regurgitation was attended. At physical examination, no changes were noted. The animal underwent endoscopy examination and simple and contrasted radiographic examinations. At the endoscopy examination, irregular lumen constriction was visualized. In the simple radiography no alterations were observed, however in the contrasted radiography with food partial stenosis and megaesophagus were observed. Based on the findings, the animal was diagnosed with megaesophagus secondary to partial esophageal stenosis and clinical treatment regarding feeding management was performed.

Keywords: Megaesophagus. Radiography. Dogs.

\section{DIAGNÓSTICO DE ESTENOSIS ESOFÁGICA PARCIAL EN UN PERRO}

\section{RESUMEN}

$\mathrm{E}$ megaesófago se define por una dilatación del esófago. Esta afección puede ser clasificada en congénita, idiopática, adquirida o secundaria. El megaesófago secundario a la estenosis se caracteriza por la obstrucción parcial o total del esófago impidiendo el paso del contenido alimentario hasta el estómago. Provoca regurgitación y pérdida de peso en el animal. Fue atendida una perra con seis años de edad, sin raza definida, con histórico de regurgitación. En el examen físico no se constataron alteraciones. El animal fue sometido a examen de endoscopia y exámenes radiográficos simples y contrastados. En el examen de endoscopia se visualizó la constricción irregular del lumen. En la radiografía simple no se observaron alteraciones, pero en la radiografía contrastada con alimento se observó estenosis parcial, y megaesófago. Con base en los hallazgos, el animal fue diagnosticado con megaesófago secundario a estenosis esofágica parcial y se procedió a tratamiento clínico debido al cuadro de la paciente.

Palabras clave: Megaesófago. Radiografía. Perros. 


\section{REFERÊNCIAS}

ADAMAMA-MORAITOU, K. K.; RALLIS, T. S.; GALATOS, A. D. Benign Esophageal stricture in the dog and cat: A retrospective study of 20 cases. Canadian Journal of Veterinary Research, v. 66, n. 1, p. 55-59, 2002.

ANDRADE, S. F. Megaesôfago secundário à miastenia grave em uma cadela da raça Pastor Alemão. Semina: Ciências Agrárias, v. 28, n. 3, p. 477-482, 2007.

BRAWNER, W. R.; BARTELS, J. E. Contrast radiography of the digestive tract. Veterinary Clinics of North America: Small Animal Practice, v. 13, n. 3, p. 599-626, 1983.

HAN, E. Diagnosis and management of reflux esophagitis. Clinical Techniques in Small Animal Practice, v. 18, n. 4, p. 231-238, 2003.

LAM, N.; WEISSE, C.; BERENT, A.; KAAE, J.; MURPHY, S.; RADLINSKY, M.; RICHTER, K.; DUNN, M.; GINGERICH, K. Begnin esophageal stenting for treatment of refractory benign esophageal strictures in dogs. Journal of Veterinary Internal Medicine, v. 27, p. 1064-1070, 2013.

SOUZA, M. G.; ZILIO, B. S.; COSTA, J. L. O. Megaesôfago em cães. Revista Científica Eletrônica de Medicina Veterinária, v. 4, n. 8, 2007. Disponível em: <http://faef.revista.inf.br/imagens_arquivos/arquivos_destaque/wuD6kj8MAEjYqeH_20135-22-17-9-14.pdf>.

TANAKA, N. M.; HOOGEVONINK, N.; TUCHOLSKI, A. P.; TRAPP, S. M.; FREHSE, M. S. Megaesôfago em cães. Revista Acadêmica: Ciência Agrária e Ambiental, v. 8, n. 3, p. 271279, 2010.

TEÓFILO, T. S.; NETO, M. O.; TORRES, M. A. O.; DIAS, H. L. M.; ALVES, G. L. Megaesôfago congênito em um cão. Veterinária e Zootecnia, v. 23, n. 4, p. 594-598, 2016.

TILLEY, L. P.; SMITH, F. W. K. In: TILLEY, L. P.; SMITH, F. W. K. Consulta Veterinária em 5 Minutos. 2. ed. Barueri: Manole, 2003. P. 942-943.

Autor para correspondência: Guilherme Albuquerque de Oliveira Cavalcanti. Campus Universitário Capão do Leão, S/N, Departamento de Clínicas Veterinárias, Faculdade de Veterinária, UFPEL. CEP 96160-000, Capão do Leão (RS). guialbuquerque@yahoo.com 